\title{
Self Interference Cancellation in Co-Time-Co- Frequency Full Duplex Cellular Communication
}

\author{
Sajjad Ali Memon ${ }^{1}$ \\ Assistant Professor in the Department of \\ Telecommunication Engineering \\ Mehran University of Engineering and Technology \\ Jamshoro, Pakistan
}

\author{
Faisal Ahmed Dahri ${ }^{2}$ \\ Postgraduate student in Department of Telecommunication \\ Engineering \\ Mehran University of Engineering and Technology \\ Jamshoro, Pakistan
}

\author{
Farzana Rauf $\mathrm{Abro}^{3}$ \\ Associate Professor in the Department of Electronics \\ Engineering \\ Mehran University of Engineering and Technology \\ Jamshoro, Pakistan
}

\author{
Faisal Karim Shaikh ${ }^{4}$ \\ Professor in the Department of \\ Telecommunication Engineering \\ Mehran University of Engineering and Technology \\ Jamshoro, Pakistan
}

\begin{abstract}
The performance of co-time co-frequency full duplex (CCDF) communication systems is limited by the selfinterference (SI), which is the result of using the same frequency for transmission and reception. However, current communication systems use separate frequencies for transmission and reception, respectively. Therefore, SI is an important issue to be fixed for future-generation systems. As the radio frequency (RF) spectrum is very scared and a CCDF system has the potential to reduce the current spectrum use by half. In this paper, a CCDF communication system is modeled and a combination of $\mathrm{RF}$ and digital cancellations is used to mitigate the SI. The simulation results reveal that the proposed combination of RF and digital cancellation achieve the bit-errorrate of $10^{-11}$ at an interference-to-signal ratio of $10 \mathrm{~dB}$, which is satisfying value for CCDF communication. The achieved efficiency of the proposed system is $13 \mathrm{bits} / \mathrm{sec} / \mathrm{Hz}$ at a signal-tonoise ratio of $50 \mathrm{~dB}$. The antenna separation of $35 \mathrm{~dB}$ is considered for the proposed model to keep the data loss as minimum as possible. The performance can be improved further by increasing digital-to-analog converter bits but with added complexity.
\end{abstract}

Keywords-Cellular; Co-Time Co-Frequency full duplex (CCFD); self-Interference cancellation (SIC); communication system

\section{INTRODUCTION}

Currently, wireless frequency spectrum turns out to be crowded and costly because of the development of bandwidthdemanding applications used in telecommunication. Thus, it is needed to present new advancements that can improve both spectrum efficiency and transmission rates. Full-duplex (FD) communication is a potential candidate technology for enhancing the spectral efficiency of next-generation wireless communication networks; such as fifth generation (5G) networks. FD cellular communication systems are also known as co-frequency co-time full duplex (CCFD), which transmit and receive signals simultaneously at the same frequency and

This work was supported by the Start-Up Research Grant Program project number 1718 of the Higher Education Commission of Pakistan. time. It improves the system performance in terms of throughput and spectral efficiency. The signal strength of selfinterference (SI) is stronger than that of the desired signal from the estimated node from 90 to $110 \mathrm{~dB}$. Therefore, with a specific goal to make FD practically implementable, canceling or dropping the interference signal to the noise floor is necessary. In the present time, there is no available electromagnetic method which can bring down SI under the noise floor. Therefore, it is necessary to further suppress the SI at the baseband stage. With the use of separate antennas, significantly increases the self-interference cancellation (SIC) level, however it contains primary disadvantage, such as multiple antennas avoid from the dense assimilation of in-band FD systems because of the required physical distance between antennas. Therefore, the system performance is declined and causes an analog to digital conversion (ADC) saturation problem [1]. The authors have demonstrated a Lab view based algorithm to deal with SIC. The video stream was used as a type of data at the central frequency of 2.2 to $2.5 \mathrm{GHz}$ with a bandwidth of $20 \mathrm{MHz}$. The ADC saturation was decreased but the overall performance of the system is not up to the mark in long-term evolution. The SIC is initiated and can be employed only on relay based node for communication. The mutual coupling model is used to cancel SIC. The power of interference was minimized to improve system performance [2]. Authors in [3] provide an overview of various concepts to decrease SIC. The measurements had been done to characterize the nature of SIC with a variety of techniques. The authors proposed the analog and digital cancellation methods but failed to provide the concluding technique. The comprehensive study is to assist in understanding and developing an appropriate cancellation technique. The researchers have mitigated the computational requirement with removing digital cancellation parameters and achieved $65 \%$ efficiency [4]. Ref. [5] personifies the potentiality, drawbacks and gaining of estimated methods. The error control coding plays a primary role in SIC to mitigate the interference. Selective filtering methods were designed to cater to the problem of SI [6]. The SIC level was controlled at some stage but not completely 
removed in all available techniques. Least mean square (LMS) algorithm and Rician channel were introduced as an interference channel with high powered in the line of sight direction. Simulations were carried out to confirm through Lab view software [7]. The receiver amplifier saturation and dynamic range were overlooked; these are considered as main aspects of canceling technique in passband signal. The researchers have extended the range of full duplex systems [811]. In present work, two antennas are required for full duplex communication; one side works as a transceiver and vice versa but the main challenge is generated through transmit antenna at the receiving antenna is addressed in such systems is a selfinterference. After 2020, higher data rate requirement with the fastest growth in data communication devices and development in mobile communication turn the idea of researchers for developing new generation networks for communication; for instance 5G. Therefore, CCFD became a latent technology and prevalent research topic in a $5 \mathrm{G}$ cellular communication system. CCFD will play a significant part in spectrum resources and efficiency. Additionally, the SIC is a core problem of CCFD so that it carries great weight and a means to do research on SIC for next-generation wireless networks and propose amendments in current cellular communication. It is always done through half-duplex; the transmission and reception have been done on distinct slots when simultaneous communication is done through FD. To boost up the spectral efficiency of cellular communication is a vital piece of research in this domain.

In this work, we have proposed the nonlinear components and antenna separations with directive antennas which enable the SIC approach. The objective of this prominent work is to make an optimal gap between transmitting and receiving antenna so that we will be able to overcome the SIC.

\section{LITERATURE REVIEW}

In recent times, lots of researchers are wasting their energies on improving the management of the spectrum efficiently. There have been a sizzling issue and many research exercises on the FD have been done for the spectral efficiency enhancement in cellular communication systems. The demand for high-speed wireless communication is burgeoning due to the rise in various multimedia services and applications. The authors have investigated the FD technology transducer along with resource management solution for cellular communication. The progress is founded on SIC technique to trim down its impact and work commercially communication transceivers. The physical spacing is considered a simple way to achieve interference cancellation [12]. In mobile communication, the frequency offset allows the distortion in orthogonality of subcarriers which results in inter-carrier interference. This entailed and proposed the coefficient which reduces the effect of interfering carriers due to the channel frequency error [13]. The oscillator phase noise limits the possibility of SIC scheme for FD. The proposed methods are applicable to estimate and cancellation phase [14]. The proliferation of wireless communication links has greatly improved to acquire high-speed broadband communication services. The femto relay node based approaches were studied based on SIC. The results draw that during the transmission of wavelength code division multiple access signals, drastically decrease in $5 \mathrm{MHz}$ including path loss and passive interference along with proper functioning of FD systems [15]. Double feed network approach is applied to mitigate the SIC and achieved 45-47 dB of SIC [16]. LMS algorithm used to overcome the echo cancellation and presented that the bandwidth of the desired data affects the system cancellation [17]. The investigators have studied simultaneous wireless information and power transfer for FD communication on the downlink. Multiple antennas were enabled for uplink and downlink communication. The presented form of results shows that their work is suitable only under restricted conditions when low data communication is needed [18]. The discrepancy between perceived spectrum and actual availability of spectrum by higher authorities of various approaches would be entertained to decrease the shortage of spectrum. Random signal architecture is persuaded to pick up the efficiency of networks due to digital domain signal cancellation [19]. The adaptive digital filters are utilized and it explores more about the low to medium ADC resolution in mixed digital cancellation. Cognitive radio needs more flexibility in spectrum than present radio systems. The authors claimed that we have exploited unutilized channel over multiple $\mathrm{GHz}$ of bandwidth spectrum. Antenna separation is presented to diminish the impact of SI [20]. Antenna design was modeled on monopole antennas in computer simulation software. The achieved results show the $50 \mathrm{~dB}$ of SI [21]. The base station and relays employ beamforming through an array of the antenna are considered for the broadband multi-cell system. The gain could be exploited for making a reduction in SI. It is experienced that with an increase in a number of antennas in picky separation may effect in SIC value [22]. The real-time and scalable digital cancellation methods were used to mitigate the SI. Video streamed with two different platforms at a central frequency of $20 \mathrm{MHz}$ of bandwidth in long-term evolution infrastructure [1]. The compatibility and capability issues had been minimized for FD communication [23]. The energy harvested from the SIC model for FD communication. During the process of downlink transmission, energy harvested for FD communication [24]. Outcomes of paper [25] present the significant improvement in energy efficiency to allow recycling of the self-energy approach. Stochastic geometry was employed to analyze the FD wireless network throughput. The performance of halfduplex was optimized based on throughput and a mathematical model has been studied which highlight the suppression of SI and suitability of FD communication.

\section{SYSTEM MODEL}

The simultaneous transmission of FD communication can be achieved through cancellation of SI. The SI signal is billion times stronger than the received signal. The initial concept of wireless communication is that radio frequency signals cannot be transmitted and received at the same time and frequency. The two nodes are attempting to transceiver simultaneously with co-time co-frequency. The interference signal is shown in the Fig. 1. Both the nodes have stronger signal at their own side from the transmitter. Such signals create interference which is known as SI. Theoretically, it is easy to solve but practically the receiver should have information of the transmitted signal and subtract it from the received signal after that decode the rest of the information signal. The model is a 
complete FD transceiver approach in terms of the component which enables to scrutinize the viability of communication channel. The block diagram of the FD is illustrated in Fig. 2. The simple architecture is considered due to its wider use in cellular communication. The digital cancellation and radio frequency cancellation module are used to process the linear operations. The ADC interface is deployed in the system to analyze the ADC bits impacts and detector input response was considered in this scenario for validation of system design. The assumption of noise figure at the receiver was discussed in form of the equation which is denoted by Frii's formula [26].

$$
F_{r x}=F_{L N A}+\frac{F_{\text {mixer }}-1}{G_{L N A}}+\frac{F_{V G A}-1}{G_{L N A} G_{\text {mixer }}}
$$

Where $F$ denotes the noise factors of different components and $G$ shows the gain of those factors. Antenna separation is a factor of RF cancellation because path losses were estimated based on the distance within the transceiver. The proper set of values of phase and amplitude would be taken for fixed delays may impact the multipath signals through coefficients and subtract interference signals from the received signal. There is a need for bulky attenuator with a fixed value to cancel required power levels for full duplex communication. The RF cancellation block requires the estimated path loss between antenna to ensure the power of reference and interference signals. The ADC interference and quantization noise can be estimated from equation 2 . The signal to quantization noise ratio can be formulated as
$S N R_{A D C}=6.02 b+4.74-P A P R$

Numbers of bits are denoted by $b$ and peak-to-average power ratio $(P A P R)$ is the fixed value considered. The clipping of the signal should be avoided based on the full range and capabilities are used. The linear processing is enabled through radio frequency and digital cancellation stages. The transmitted signal from antenna attenuated from the power amplifier. The input ADC and data input are considered as an interface in the system. The ADC input is controlled by the automatic gain control component at a constant level. Cancellation signal has high power than the desired signal contains a reduced power level. The dynamic range of ADC bits is preserved would be actuated through SI to impact at the minimum level of the desired signal.

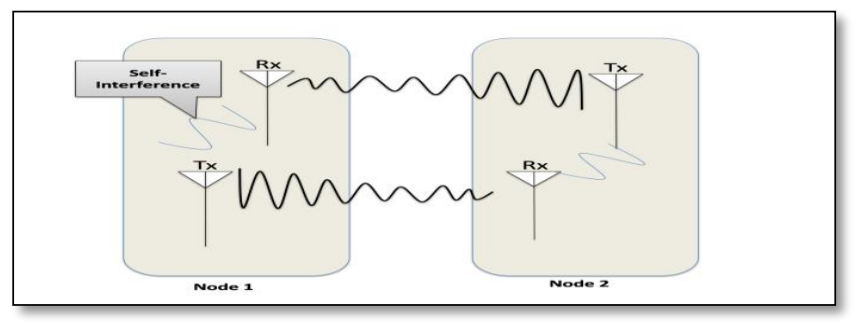

Fig. 1. The Diagram of SI from the Own Transmitter Device at the Receiver.

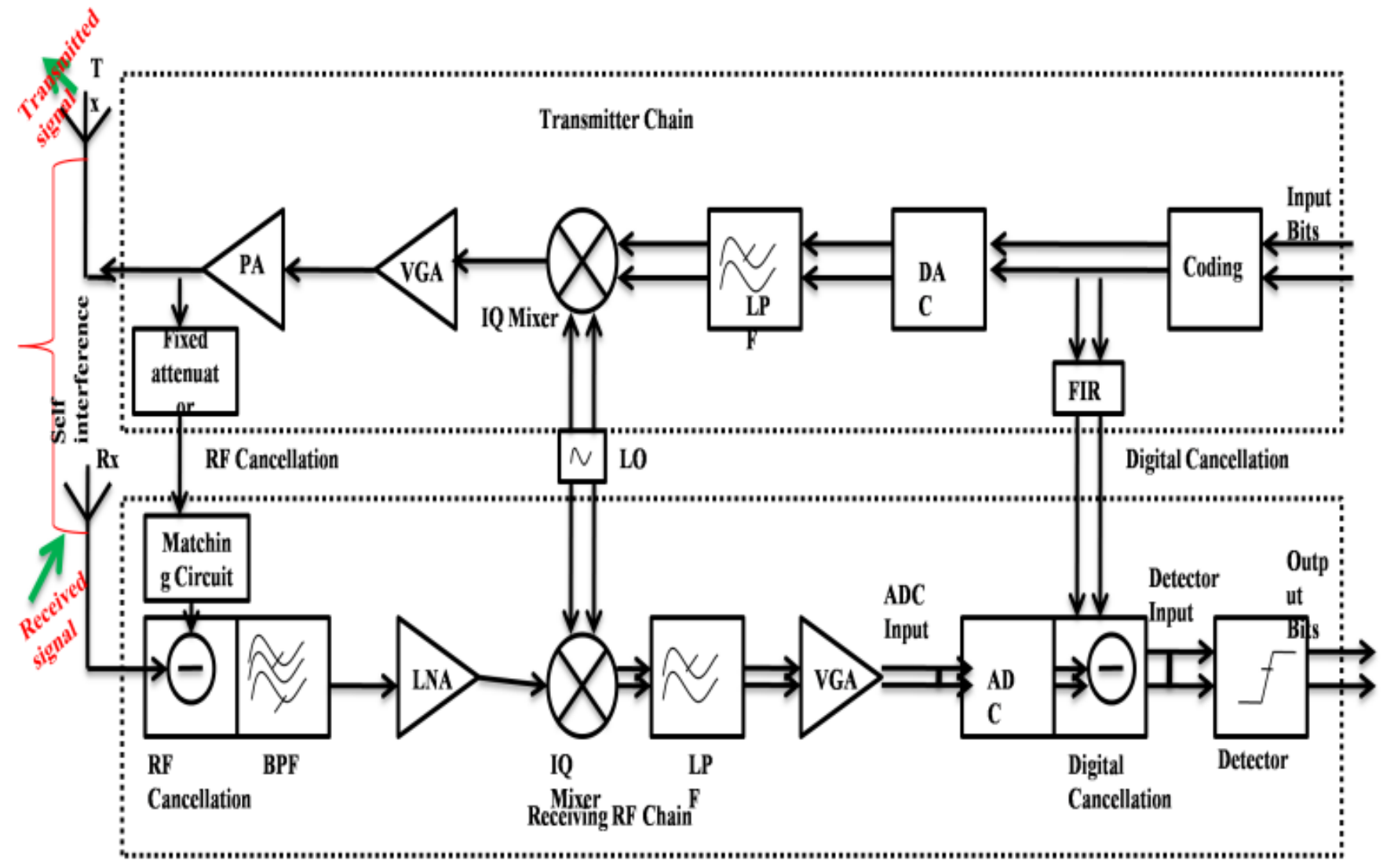

Fig. 2. The Simulated Design for FD Transceiver Framework Subjects to Desired and SI Signals [27]. 


\section{SimUlation RESUlts \& DisCUSSION}

The section provides the details regarding the performance of simulated and proposed SIC scheme under different conditions. Matlab 2017a is used to simulate the system. The system is operated in FD mode, where simultaneous transmission and reception take place at the same time and frequency. The entire communication chain of transmitter and receiver is modeled to implement analog and digital cancellation. Fig. 3 presents the performance of the proposed design in terms of symbol error rate with the number of symbol to noise rate and theoretical symbol error rate (SER). The digital to analog conversion (DAC) impact on a different number of bits was analyzed for the SI system. Fig. 4 shows the analog to the digital impact of bits at the different range was investigated. In this system, SER values are optimal to reduce the SI. The increase in bits can provide better outcome as illustrated in the results. The presented forms of results are under assumptions that radio cancellation is considered as reference signal after the power amplifier. The power amplifier introduces nonlinear distortion in the RF cancellation of the transmitter chain. It is shown in the above figure that the number of DAC bits increased to enhance the performance while ADC bits are taken as fixed. It is noted that the result does not infer that more accuracy can be added to the system by increasing only the DAC bits. The word length of the system is dependent on the DAC bits and floating points are trimmed to set estimated constant quantization levels. The SI and desired channels were estimated based on fixed DAC bits with five training symbols. It is visible in Fig. 4 that performance is irrelevant as a contrast with theoretical. The performance improves with increasing the number of ADC bits and recommended that to quantify the bits in $\mathrm{ADC}$ is not a bottleneck. The overlapping is founded in an ADC bit sequence from 12 to 16 . The lost bits increased as shown in Fig. 5 because of degradation in radio cancellation ability, it implies the SI power is high at the interface of ADC. The need for ADC is very much increased for achieving SI cancellation at the analog domain. It is observed that roughly 6 bits lost because of SI with greater transmit power of $25 \mathrm{dBm}$. This accentuates the way that in this situation SI is restricting factor for power. This show higher power is used which requires a number of ADC bits to reduce the loss of bits. The performance of bit error rate (BER) of the proposed scheme is presented in Fig. 6 in which nonlinear distortion beats the conventional schemes. The $10 \mathrm{~dB}$ of the gain of interference signal rate was accomplished with the proposed method. The $K$ is a Rician channel factor at $K=3$, achieved better results and suitable for cellular communication. Fig. 7 depicts the spectral efficiency with no distortion suppression along half and full duplex results based on SNR values. The half-duplex system is restricted from the noises such as Gaussian; the SNR is characterized through the desired signal power divided by receiver Gaussian noise power. The better results of half and full duplex cellular communication can be achieved with high SNR values.

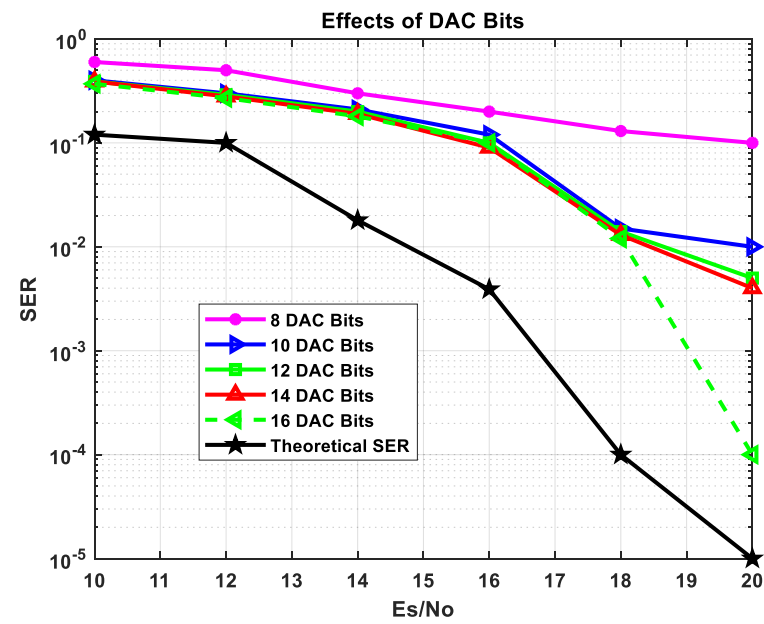

Fig. 3. Effects of Different DAC Bits on Symbol Error Rate for FD Communication.

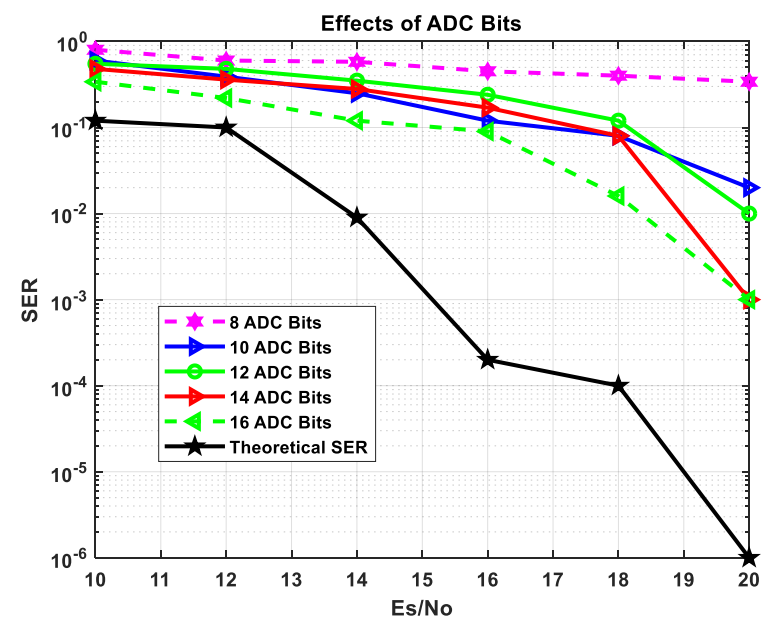

Fig. 4. Effects of Different ADC Bits on SER with a Fixed Value of DAC for FD Communication.

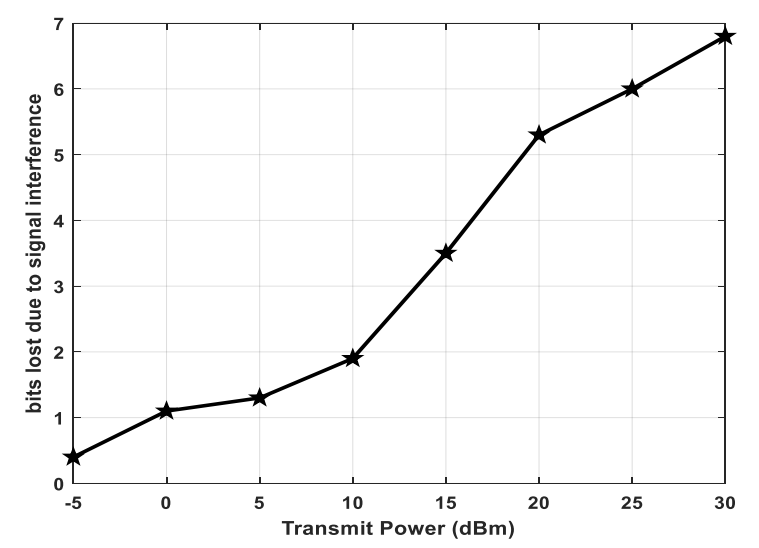

Fig. 5. Bits Lost Due to the SI Versus Transmit Power At Fixed Values of ADC with $35 \mathrm{~dB}$ Antenna Separation. 


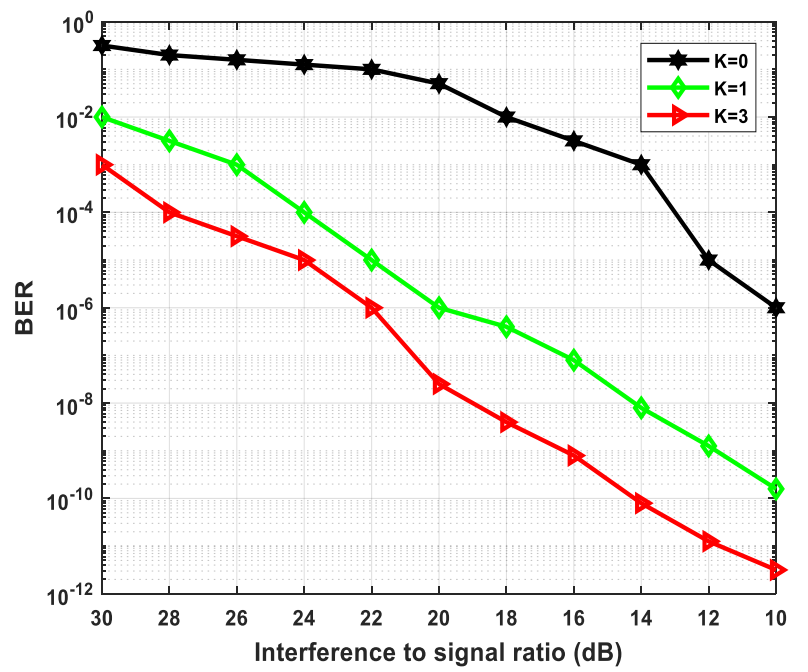

Fig. 6. BER Versus ISR with Different Rician Channel Order $(K=0,1,2)$ for FD Communication.

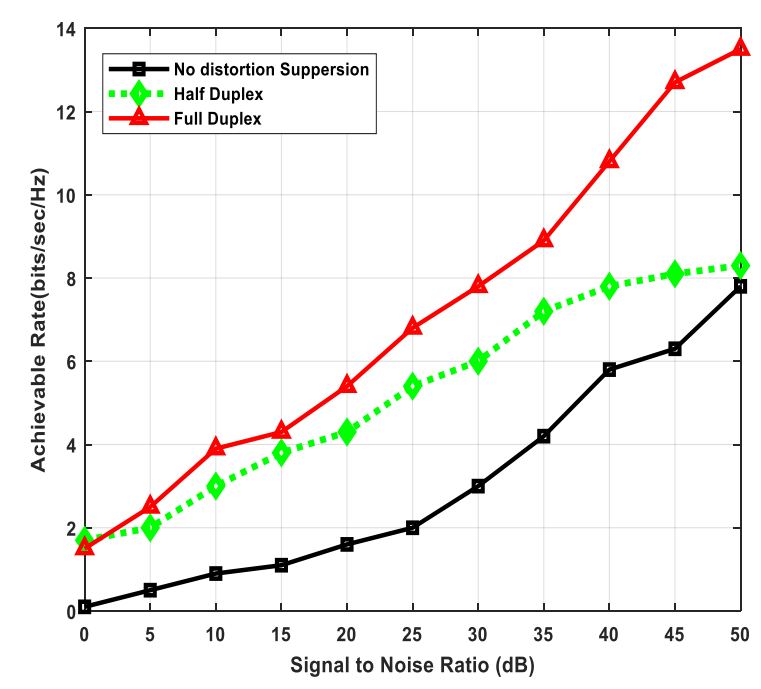

Fig. 7. Outcomes of Achievable Rate Versus SNR for a Half, No Distortion Suppression and FD Communication at Specific SI Signal Strength Values.

\section{CONCLUSION}

In this work, the SIC scheme is proposed for the CCFD communication system. This approach mitigates the SI and enables FD communication based on optimal and effective results. It is observed that high SNR would improve the performance of FD. The non-linearity impact is reduced and accumulated good outcomes from the simulated design. The system reliability is validated by comparing the spectral efficiency of FD and half duplex communication system. Quantization noise limits the tolerance in terms of transmitting power for the receiver ADC converter and digital cancellation. This issue is mitigated and achieved good results. The achieved results are improved as compared to existing works. The presented results signify that proposed method mitigate interference signal and compared to a conventional half-duplex system. To tune the SNR value, the performance of FD is maximized. The achievable rates of no distortion suppression, half duplex, and $\mathrm{FD}$ are 7,8 and $13 \mathrm{bits} / \mathrm{sec} / \mathrm{Hz}$ attained, respectively. The minimum BER is accomplished through the proposed method and performance is suitable for CCFD cellular communication.

\section{REFERENCES}

[1] Chu, Jian-Ya, Yuan-Te Liao, Chun-Yi Hung, and Terng-Yin Hsu. "Implementation of digital self-interference cancellation in LTE-based CCFD transmission." In Dependable and Secure Computing, 2017 IEEE Conference on, pp. 475-476. IEEE, 2017.

[2] Pawinee Meerasri, Peerapong Uthansakul, and Monthippa Uthansakul, "Self-Interference Cancellation-Based Mutual-Coupling Model for FullDuplex Single-Channel MIMO Systems," International Journal of Antennas and Propagation, vol. 2014, Article ID 405487, 10 pages, 2014. https://doi.org/10.1155/2014/405487.

[3] Le-Ngoc, Tho, and Ahmed Masmoudi. "Self-Interference-Cancellation in Full-Duplex Systems." In Full-Duplex Wireless Communications Systems, pp. 13-25. Springer, Cham, 2017.

[4] Korpi, Dani, Lauri Anttila, and Mikko Valkama. "Nonlinear selfinterference cancellation in MIMO full-duplex transceivers under crosstalk." EURASIP Journal on Wireless Communications and Networking 2017.1 (2017): 24.

[5] Fu, Tong. Full-duplex Wireless Communications: Capacity Analysis, Low-density Parity-check Coding and Feasibility. Diss. McGill University Libraries, 2015.

[6] Öncel, Aksay Fatih. On Self-Interference Cancellation in Wireless Full duplex, Diss. Middle East Technical University, 2016.

[7] Koirala, Kumud. Adaptive Self-Interference Cancellation in Full-Duplex Radio. Tampere University of Technology (2016).

[8] Krier, John R., and Ian F. Akyildiz. "Active self-interference cancellation of passband signals using gradient descent." In Personal Indoor and Mobile Radio Communications (PIMRC), 2013 IEEE 24th International Symposium on, pp. 1212-1216. IEEE, 2013.

[9] $\mathrm{Li}, \mathrm{Na}$, Weihong $\mathrm{Zhu}$, and Haihua Han. "Digital interference cancellation in single channel, full duplex wireless communication." In Wireless Communications, Networking and Mobile Computing (WiCOM), 2012 8th International Conference on, pp. 1-4. IEEE, 2012.

[10] Shen, Ying, Juan Zhou, and Youxi Tang. "Digital Self-Interference Cancellation in Wireless Co-time and Co-frequency Full-Duplex System." Wireless Personal Communications 82, no. 4 (2015): 25572565.

[11] Li, Jiong, Hang Zhang, and Menglan Fan. "Digital self-interference cancellation based on independent component analysis for co-time cofrequency full-duplex communication systems." IEEE Access 5 (2017): 10222-10231.

[12] Kari Rikkinen, Alok Sethi, Visa Tapio , Laura González ,Cristina Lavín, Reinel Marante ,Björn Debaillie, Mina Mikhael, Hans Suys,Barend van Liempd, Mir Ghoraishi, Hassan Malik . "Full-Duplex Radios for Local Access - DUPLO” Ref. Ares(2015)2277009 - 2015.

[13] Zhao, Yuping, and S-G. Haggman. "Intercarrier interference selfcancellation scheme for OFDM mobile communication systems." IEEE Transactions on Communications 49, no. 7 (2001): 1185-1191.

[14] Ahmed, Elsayed, Ahmed M. Eltawil, and Ashutosh Sabharwal. "Selfinterference cancellation with phase noise induced ICI suppression for full-duplex systems." In Global Communications Conference (GLOBECOM), 2013 IEEE, pp. 3384-3388. IEEE, 2013.

[15] Akyildiz, Ian F., Elias Chavarria-Reyes, David M. Gutierrez-Estevez, Ravikumar Balakrishnan, and John R. Krier. "Enabling next generation small cells through femtorelays." Physical Communication 9 (2013): pp. $1-15$.

[16] An, Changyoung, and Heung-Gyoon Ryu. "Double Balanced Feed Network for the Self-Interference Cancellation in Full Duplex Communication System." Wireless Personal Communications 92, no. 4 (2017): 1599-1610.

[17] Avasarala, Sanjay. "Adaptive Baseband Interference Cancellation for Full Duplex Wireless Communication Systems". Diss. Arizona State University, 2016. 
[18] Chen, Dong-Hua, and Yu-Cheng He. "Full-Duplex Secure Communications in Cellular Networks with Downlink Wireless Power Transfer." IEEE Transactions on Communications 66.1 (2018): 265-277.

[19] Yang, Jing. "Time domain interference cancellation for cognitive radios and future wireless systems". Diss. UC Berkeley, 2010.

[20] Jasim, A. A., K. M. Younus, A. Ali, K. H. Sayidmarie, A. Alhaddad, and R. A. Abd-Alhameed. "A simple self-interference cancellation technique for full duplex communication." In Internet Technologies and Applications (ITA), 2017, pp. 224-229. IEEE, 2017.

[21] Larsson, Peter, and Mikael Prytz. "MIMO on-frequency repeater with self-interference cancellation and mitigation." In Vehicular Technology Conference, 2009. VTC Spring 2009. IEEE 69th, pp. 1-5. IEEE, 2009.

[22] Zarbouti, Dimitra, George Tsoulos, and Georgia Athanasiadou. "Effects of antenna array characteristics on in-band full-duplex relays for broadband cellular communications." ICT Express 1, no. 3 (2015): 121 126.
[23] Sim, Min Soo, Kwang Soon Kim, and Chan-Byoung Chae. "SelfInterference Cancellation for LTE-Compatible Full-Duplex Systems." In 2018 IEEE International Conference on Communications Workshops (ICC Workshops), pp. 1-6. IEEE, 2018.

[24] Yadav, Animesh, Octavia A. Dobre, and H. Vincent Poor. "Is SelfInterference in Full-Duplex Communications a Foe or a Friend?." IEEE Signal Processing Letters 25, no. 7 (2018): 951-955.

[25] Z. Tong and M. Haenggi, "Throughput Analysis for Full-Duplex Wireless Networks With Imperfect Self-Interference Cancellation," in IEEE Transactions on Communications, vol. 63, no. 11, pp. 44904500, Nov. 2015.

[26] Gu, Qizheng. RF system design of transceivers for wireless communications. Springer Science \& Business Media, 2005.

[27] Korpi, Dani, et al. "Full-duplex transceiver system calculations: Analysis of ADC and linearity challenges." IEEE Transactions on Wireless Communications 13.7 (2014): 3821-3836. 